\title{
Predicting Acute Kidney Injury Following Open Partial Nephrectomy Treatment Using SAT-Pruned Explainable Machine Learning Model
}

Teddy Lazebnik ( $\nabla$ t.lazebnik@ucl.ac.uk)

University College London https://orcid.org/0000-0002-7851-8147

\section{Zaher Bahouth}

Bnei Zion Hospital

Svetlana Bunimovich-Mendrazitsky

Ariel University

Sarel Halachmi

Bnei Zion Hospital

\section{Research Article}

Keywords: AKI prediction, SAT pruned random forest, PN treatment complication prediction

Posted Date: February 18th, 2022

DOI: https://doi.org/10.21203/rs.3.rs-737087/v1

License: (c) (i) This work is licensed under a Creative Commons Attribution 4.0 International License. Read Full License 


\title{
Predicting Acute Kidney Injury Following Open Partial Nephrectomy Treatment Using SAT-Pruned Explainable Machine Learning Model
}

\author{
Teddy Lazebnik ${ }^{1 * x}$, Zaher Bahouth ${ }^{2 *}$, Svetlana Bunimovich- \\ Mendrazitsky 3 , Sarel Halachmi 2 \\ University College London, Cancer Institute, Department of Cancer Biology 1 \\ Bnai Zion Medical Center, department of Urology 2 \\ Ariel University, department of Mathematics 3 \\ Authors contributed equally \\ Corresponding author: t.lazebnik@ucl.ac.uk $x$
}

\begin{abstract}
Background: One of the most devastating complications of Partial Nephrectomy (PN) is Acute Kidney Injury (AKI), which could have a negative impact on subsequent renal function and occurs in up to $24.3 \backslash \%$ of patients undergoing PN. The aim of this study was to predict the occurrence of AKI following PN using preoperative parameters by applying machine learning algorithms.

Methods: We included all adult patients $(n=723)$ who underwent open PN in our department since 1995 and on whom we have data on the pre-operative renal function. We

developed a random forest (RF) model with Boolean satisfaction-based pruned decision trees for binary classification (AKI or non-AKI). Hyper-parameter grid search was performed to optimize the model's performance. Five-fold cross-validation was applied to evaluate the model. We implemented a RF model with greedy feature selection to binary classify AKI and non-AKI cases based on pre-operative data.

Results: The best model obtained a 0.69 precision and 0.69 recall in classifying the AKI and non-AKI groups on average $(k=5)$. In addition, the model's probability to correctly classify a new prediction is 0.75 . The proposed model is available as an online calculator.

Conclusions: Our model predicts the occurrence of AKI following open PN with (75\%) accuracy. We plan to externally validate this model and modify it to minimally-invasive PN.
\end{abstract}

Keywords: AKI prediction; SAT pruned random forest; PN treatment complication prediction. 
Renal cell carcinoma (RCC) represents about $3 \%$ of all cancer-related cases in 2018 , with the

highest incidence occurring in Western countries (1). During the last decades, stage migration towards localized disease has occurred (2). Partial nephrectomy (PN) is the treatment of choice for localized cT1 renal masses (3). The main advantage of PN is the preservation of renal function compared to radical nephrectomy (4). One of the adverse effects of PN is post-operative acute kidney injury (AKI), which increases the risk of long-

term chronic kidney disease (CKD) with its consequences, including decreased overall survival (5), although some studies questioned its impact on long-term renal function (6). The prevalence of AKI following PN is reported to be up around $25 \%$ and is dependent on surgical approach, patient baseline characteristics, and the definition of AKI used in each study (7).

Machine learning (ML) based models which predict different clinical properties have been shown to be a useful tool (8) and particularly in clinical practice (9). ML models can be classified into three main subtypes: classification, search, and prediction. In this paper, we focus on the latter in order to predict AKI following PN. Prediction ML models provided with retrospective data are able to find complex statistical connections between different parameters (this step is usually referred to as the learning process) (8). As a result, upon providing a new set of parameters, these models are able to predict, with fair accuracy, the

outcome one wishes to retrieve. Weng et al. (10) used four ML algorithms to predict cardiovascular risk, showing improvement of all four compared to standard algorithms. A study by Wu et al. (11) developed an ML model to predict fatty liver disease. The authors

used five different ML algorithms on the same data, where RF showed the best results. Specifically, previous studies investigating the performance of ML models in predicting AKI have yielded promising results $(12 ; 14)$. However, the accuracy of these models is not optimal, and we thought that using an ML model, we can increase the accuracy of these models. In this study, we aim to apply an explainable ML model to predict AKI in patients undergoing open PN. We hypothesized that ML models could identify and learn from pre-operative parameters and predict the AKI outcome. A self-explainable prediction system that is based on ML was then built and deployed online. A schematic view of the workflow of the proposed framework is shown in Fig. 1.

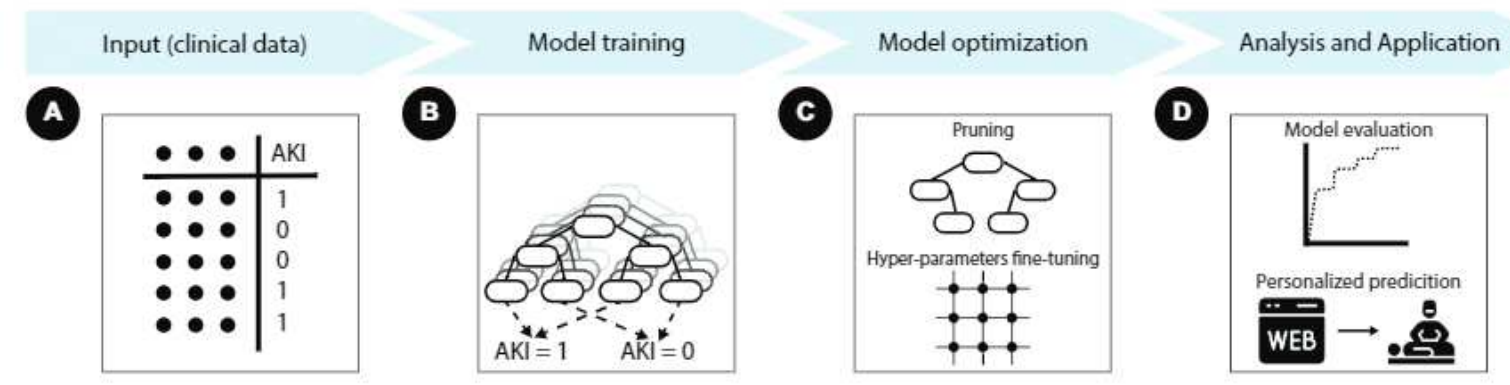

Figure 1: A workflow of the proposed framework. 


\subsection{Data acquisition}

Since 1995, we have been continuously extending our open PN database to include surgical and oncological parameters. For this particular study, we included all adult ( $>18$ years) patients who underwent open PN for enhancing solid renal mass and then split the data into AKI and non-AKI. Patients with a solitary kidney or multiple tumors were excluded from this study. Therefore, the PN database includes 723 patients. Renal function was assessed the day before surgery, on the day of the surgery, and on a daily basis after the surgery until discharge which more often than not was on post-operative day 3.

\subsection{Operative Technique}

An extraperitoneal, extrapleural supra-11th rib incision was done on the operated side. IV Mannitol was given before clamping the renal artery. In situ renal hypothermia was done by cooling the surface of the kidney with ice slush for 10 to 15 minutes immediately after clamping the renal artery. The tumor was enucleated with a minimal rim of normal parenchyma. Renorrhaphy was done using either 2/0 VICRYL interrupted sutures or tissue adhesive BioGlue (CryoLife, Atlanta, GA). A more detailed surgical technique has been previously published by our group (17).

\subsection{Renal Function Assessment}

Baseline serum Creatinine ( $\mathrm{sCr}$ ) was measured the day before surgery. We used both the RIFLE (risk, injury, failure, loss of kidney function, and end-stage renal failure) (15) and AKIN

(Acute Kidney Injury Network) (16) criteria to define AKI, comparing each of the postoperative renal function assessments to the baseline level. AKI was defined as the occurrence of one of the following conditions: 1 ) an increase in serum Creatinine of $\geq 0.5$ times above baseline in the first week following surgery, 2) an increase in $\mathrm{sCr}$ by $\geq 0.3 \mathrm{mg} / \mathrm{dl}(\geq 26.5 \mathrm{mmol} / \mathrm{l})$ above baseline in the 48 hours window post-operatively, or 3 ) reduction of more than 25 percent of the estimated Glomerular Filtration rate in the 7 days period after surgery. In total, 231 patients developed AKI based on the aforementioned criteria and constituted the AKI group, and 492 did not develop AKI and therefore were classified as non-AKI. 723 patients is considered a large enough set to use for the methods shown in the following sections (18).

\subsection{Data split}

In order to develop ML algorithm, the study population was compiled into a data set, split into a training cohort from which the proposed algorithm was derived and a validation cohort on which the model was applied and tested. The training cohort was derived from a random sampling of $80 \%$ of the data set, and the validation cohort comprised the remaining $20 \%$. The division process was repeated 1000 times looking for the optimal split that 
ensures no statistically significant differences between the two cohorts in demographics or AKI outcome. The split was carried on such that the divisions are minimizing the differences of the age, gender, smoking years, and AKI parameters in both the training and validation cohorts. The distribution of the parameters age, smocking, gender, and AKI in both these cohorts are shown in Eq. (1).

$\left[\begin{array}{ccc}\text { Parameter } & \text { Training Cohort } & \text { Validation Cohort } \\ \text { Age } & 61.23 \pm 12.05 & 60.92 \pm 13.14 \\ \text { Smoking } & 17.61 \% \pm 38.15 \% & 18.34 \% \pm 38.74 \% \\ \text { Gender } & \text { Male }: 37.98 \%, \text { Female }: 62.02 \% & \text { Male }: 35.45 \%, \text { Female }: 64.55 \% \\ \text { AKI } & 46.84 \% \text { with AKI } & 38.54 \% \text { with AKI }\end{array}\right]$

\subsection{Algorithm}

We used the random forest (RF) ML algorithm (19). We selected the RF algorithm because it can provide a simple explanation of the model's prediction to healthcare professionals while obtaining a good accuracy on a relatively small data set (20). We applied the proposed binary AKI prediction decision tree (DT) algorithm on the training cohort and then validated it on the validation cohort that was completed using the sklearn library with Python 3.5. The model's hyper parameters were determined using the grid search method (21) (see Section 2.9) and five-fold cross-validation on the training cohort to determine the values which led to the best performance.

\subsection{Feature selection}

We performed a feature selection in the following order: first, we manually filtered the features available before the surgery (marked as $F$ ). Afterward, we evaluated the model's

accuracy, picking one feature from $F$. The feature that resulted in the model's highest accuracy was chosen $F_{1}$. Then, an additional feature from the remaining feature set $\left(F \backslash F_{1}\right)$ was added to the chosen feature set from the previous step such that the model's accuracy was the highest between all combinations. The process was repeated until the gain in the model's accuracy upon adding a new feature became less than $1 \%$.

\subsection{Model pruning}

After training the model, we transformed each DT in the RF into a respective Boolean satisfaction problem (SAT). Each branch was converted into a Boolean condition $r: x_{1}$ $\wedge x_{2} \wedge \cdots \wedge x_{n}$ where $\left\{x_{i}\right\}_{i=1}^{n}$ where the conditions in each node in the branch and $r$ was the result label node. Branches with the same result label $r$ were stitched together using the 'or' logical operator (V). Afterward, each Boolean condition was reduced to the minimal Boolean condition that satisfied the same inputs. The result of Boolean condition was converted back into a DT. 


\subsection{Statistical analysis}

We performed a five-fold cross-validation to evaluate the model's accuracy. The data was divided into five cohorts where four cohorts were used for the training cohort and one for the testing cohort. The process was repeated five times, allowing each patient to be included in both the training and test cohorts. The receiver operating characteristic (ROC) curve was used to measure the model's classification ability. At each point, the recall and precision were presented in correspondence with a specific decision threshold. The area under the ROC curve (AUC) was used to quantify the model's classification ability. Finally, the importance of each feature depended on the reduction of classification accuracy caused by removing the feature (e.g., information gain) (22).

\subsection{Hyper-parameter fine-tuning}

We performed hyper-parameter fine-tuning using the grid search method, based on the model's accuracy (21). The grid search was performed on

$$
\mathbb{H}:=[\text { depth }, M S P L, L C, n],
$$

Where depth is an individual DT tree depth; MSPL is the minimal number of samples for a leaf; $L C$ is the leaf count; and $n$ is the number of trees in the RF model.

\section{Results}

\subsection{Decision features}

Implementation of the method described in Section 2.6 on 31 features (see Appendix) resulted in a set of eight features

$$
F:=[\text { size, renal, age, baseHB, WIT, weight, height, creatinine }],
$$

Where size is the size of the tumor in centimeters; renal is the RENAL score; age is the patient's age in years at the time of the surgery; baseHB is the baseline hemoglobin in $\mathrm{g} / \mathrm{dL}$; WIT is the warm ischemia time in minutes; weight is the patient's weight in kilograms at the time of the surgery; height is the patient's height in centimeters at the time of the surgery; and creatinine is the baseline pre-operative Creatinine in $\mathrm{mg} / \mathrm{dL}$. The model found that WIT contributed significantly to the accuracy of the model. However, WIT is surgical parameter, and is not available beforehand. In order to overcome this, we defined a feature called

$W_{I T}^{*}$ which is an estimate of the real WIT. The WIT* is obtained using the k-nearest neighbors (KNN) algorithm (where $k=5$ and the distance metric is weighted by distance and the average is obtained using the grid search method) on the other seven features which are available before the surgery. To evaluate the quality of the $W I T^{*}$ feature compared to 
the original WIT feature, we performed a five-fold test on the WIT feature with the KNN algorithm. A linear regression on the values $\left(W I T, W I T^{*}\right)$ was obtained, resulting in a coefficient of determination $\left(R^{2}\right)$ of 0.879 . Namely, the $W I T^{*}$ feature well estimate the

WIT feature and therefore, we wereable to replace the feature space to:

$$
F^{*}:=[\text { size, renal, age, baseHB, WIT* , weight, height, creatinine }] .
$$

A Pearson correlation coefficient between all pairs of $F$ was then obtained, showing a 0.57 correlation between the renal and size features and 0.45 correlation between patient height and weight. The first correlation is trivial as the renal score includes the size. In addition, the

second correlation is already reported in other studies (25). All other combinations of features from $F$ have absolute correlation below 0.3 , supporting the fact that the featurespace is mostly linearly independent

\subsection{AKI classification model}

We trained the RF model (see Section 2.5) on the clinical data set as described in Section 2.4 on the feature set $F$. Afterward, we performed hyper-parameter fine-tuning as described in

Section 2.9. Then, we carried out pruning on the best model (see Section 2.7). As a result, we obtained a model with 107 DTs; each one of these DTs had up to five levels of depth. The number of leaves is different for each tree in the RF due to the pruning process. The model was validated on the validation set using five-fold cross-validation analysis. The precision obtained $0.69 \pm 0.085$. Similarly, the recall obtained $0.69 \pm 0.062$. The features' importance is presented in Table 1.

\begin{tabular}{|l|c|c|c|c|c|c|c|c|c|}
\hline Feature & Size & renal & age & BaseHB & weight & height & creatinine & WIT & WIT* \\
\hline $\begin{array}{l}\text { Original } \\
\text { Importance }\end{array}$ & 0.13 & 0.11 & 0.17 & 0.05 & 0.15 & 0.05 & 0.21 & 0.13 & 0 \\
\hline $\begin{array}{l}\text { Estimated } \\
\text { Importance }\end{array}$ & 0.14 & 0.11 & 0.19 & 0.06 & 0.12 & 0.03 & 0.23 & 0 & 0.12 \\
\hline $\begin{array}{l}\text { Core } \\
\text { Importance }\end{array}$ & 0.17 & 0.11 & 0.2 & 0.06 & 0.16 & 0.06 & 0.24 & 0 & 0 \\
\hline
\end{tabular}

Table 1: Model's features importance. The original importance is the one obtained by a model that uses WIT. The estimated importance is the one obtained by estimating WIT (e.g., $W I T^{*}$ using the other seven features). The core importance is the importance of the seven features as the weighted average in contribution to the final prediction of $W I T^{*}$.

Furthermore, we derived the ROC curve of the model, as shown in Fig. 2. The AUC was found to be 0.75 . In addition, the average confusion matrix was:

$$
\left[\begin{array}{ccc} 
& \text { True } & \text { False } \\
\text { Positive } & 0.34 & 0.41 \\
\text { Negative } & 0.07 & 0.18
\end{array}\right]
$$




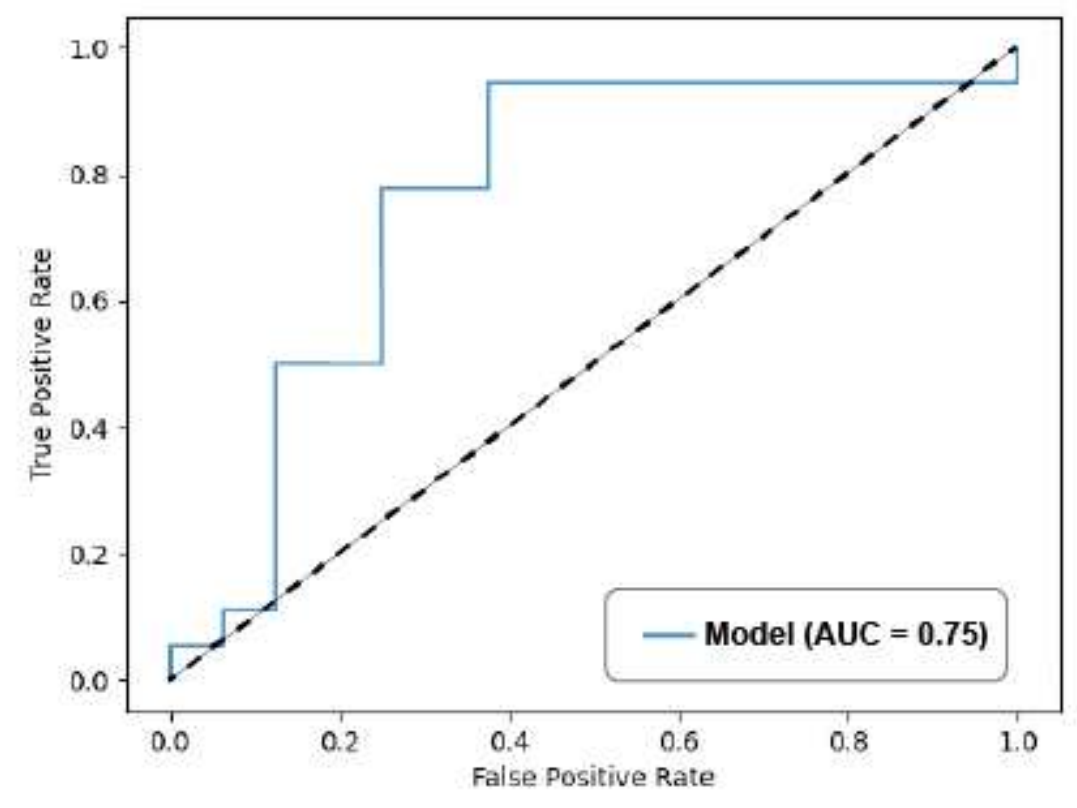

Figure 2: The ROC curve of the model's prediction on the binary AKI classification, with AUC

of 0.75 .

\subsection{Interface}

The model has been deployed as a web service ${ }^{1}$. Fig. 3 shows the model's interface as a web

service.

\begin{tabular}{|c|c|c|}
\hline Size & renal & age \\
\hline 1 & 1 & 1 \\
\hline Base $\mathrm{Hb}$ & WIT* & creatinine \\
\hline 1 & 1 & 1 \\
\hline weight & height & \\
\hline 1 & 1 & \\
\hline
\end{tabular}

The prediction is: o with $(55.2 \%$ confidence).

Because:

creatinine $<=1.71$ and

renal $<=9.5$ and

Base Hb $<=14.05$ and

weight $<=100.5$

Figure 3: The model's interface and prediction as a web service. The user inserts a patient's data into the form and by clicking on the predict button obtains the AKI binary prediction 
with the model's confidence. In addition, an explanation of the model's prediction is provided below.

\section{Discussion}

AKI following PN is a unique entity, which significantly differs from medical and post-surgical $\mathrm{AKI}$; in addition to the common risk factors for medical AKI, patients undergoing PN have increased risk for AKI due to the associated blood loss and relative hypovolemia and, more importantly, the clamping of the renal artery and the loss of functional tissue. Several studies reported an increased risk of chronic kidney disease (and mortality) in patients who develop AKI (23). The incidence of AKI following PN varies depending on several parameters, including surgical approach, the definition used for AKI, and the cohort reported in each

study. In a recent study by Tachibana et al. (24), the authors reported less than $11 \%$ AKI following robotic PN and almost 50\%incidence following open PN (24). Our results demonstrate that the development of AKI following PN can be accurately predicted based only on clinical information routinely collected before surgery. The proposed models performed well according to all evaluation criteria and achieved a higher AUC and accuracy score compared to the classical scoring methods. The proposed model has similar scores to the modern ML-based models (12) and AUC in accordance with previous large studies on general intensive-care unit patients developing medical AKI (27). We used a RF model (as an

ensemble of DT models) as the ML algorithm for our model in order to take advantage of the explainable property of this model. In addition, by using the SAT pruning algorithm, we were able to obtain the shortest, and therefore, easiest to understand explanation for each

prediction. This explanation provides the treating Urologist with the ability to agree or disagree with the model on unique cases and has a second validation process on the model's prediction based on the personnel's wider understanding of the patient's condition (i.e., man-in-the-loop) (29). The proposed AKI prediction model could be publicly available as an online prognostic calculator, providing a platform for future AKI-prediction studies, and complementing existing risk assessment scores (30). One could argue that a better endpoint would be the risk of CKD following PN, which is the most important endpoint. In this study, we aimed to predict AKI as it was demonstrated to increase the risk for CKD. A model that predicts CKD is harder to build, and this is one of our future projects. The main limitation of our study is being an open PN cohort, and it is yet to be determined if it will apply to patients who undergo minimally invasive surgery. The second limitation is the relatively small cohort for this model, although others used smaller cohorts and reported good results (18). Another limitation is its retrospective nature and subsequently some missing data. Moreover, we used an estimated parameter, warm ischemia time, to predict the AKI based on pre-operative parameters. However, despite these limitations, our model can predict AKI with relatively high accuracy (75\%). In conclusion, our ML model can reliably predict AKI following open PN. Future possible research is to extend the size of the database and perform stability analysis for controversial cases in order to improve the robustness of the proposed model. 


\section{Conclusion}

The usage of an explainable machine learning-based model in predicting acute kidney injury following partial nephrectomy treatment can help clinicians to be better prepared for complications after the surgery while reducing resource spending by better predicting patients that may suffer these complications.

\section{Appendix}

Clinical parameters. The data set used in this research is described in Table S1.

\begin{tabular}{|l|l|}
\hline Feature & Description \\
\hline Side & Side of the tumor - left or right. \\
\hline Location & The location of the tumor - upper, middle, lower, hilar. \\
\hline Endophytic & Binary existence of endophytic. \\
\hline Thmor size & Tumor size in centimeters. \\
\hline RENAL score & The renal score between 4 and 12. \\
\hline cT stage & Based on pre-operative imaging. \\
\hline pT stage & Pathological T stage. \\
\hline pN stage & Pathological N stage. \\
\hline pM stage & Pathological M stage. \\
\hline Subtype & Renal cell carcinoma, non-RCC cancer, benign. \\
\hline Furhman grade & Furhman grade between 1 and 4. \\
\hline Sinus fat invasion & Binary existence of Sinus fat invasion. \\
\hline Baseline Creatinine & Pre-operative serum Creatinine levels (mg/dL). \\
\hline Baseline eGFR & Pre-operative eGFR \\
\hline ps Creatinine & Highest post-operative serum Creatinine (mg/dL). \\
\hline ps eGFR & Worst post-operative eGFR. \\
\hline Smoking & Years of active smoking. \\
\hline Race & Caucasian, afro-israeli. \\
\hline HgdtCr & Highest increase in post-operative serum Creatinine. \\
\hline LwDteGFR & Worst decrease in post-operative serum Creatinine. \\
\hline Age & in years \\
\hline BaseHb & Baseline Hemoglobin (g/dL). \\
\hline Ischemia Time & Ischemia time in minutes. \\
\hline EBL & Estimated blood loss (ml). \\
\hline $\begin{array}{l}\text { Ischemic heart dis- } \\
\text { ease }\end{array}$ & Binary existence of ischemic heart disease. \\
\hline Diabetes & Binary existence of diabetes. \\
\hline Gender & Patient's gender (Male or Female). \\
\hline Weight & Patient's weight in kilograms. \\
\hline Height & Batient's height in centimeters. \\
\hline AKI & \\
\hline
\end{tabular}

Table S1: Data set feature names and descriptions used in the research. 
Training-Validation Cohorts Division. The data set is divided into training and validation cohorts with $80 \%$ and $20 \%$, respectively. The distribution of the parameters age, smoking, gender, AKI, Baseline creatinine, Baseline GFR, Post operative creatinine, and Post operative creatinine in both these cohorts are shown in Table S2.

\begin{tabular}{|l|c|c|}
\hline Parameter & Training Cohort & Validation Cohort \\
\hline Age (years) & $61.23 \pm 12.05$ & $60.92 \pm 13.14$ \\
\hline smoking & $17.61 \% \pm 38.15 \%$ & $18.34 \% \pm 38.74 \%$ \\
\hline Gender & Male: $37.98 \%$, Female: $62.02 \%$ & Male: $35.45 \%$, Female: $64.55 \%$ \\
\hline AKI & $46.84 \%$ with AKI & $38.54 \%$ with AKI \\
\hline Baseline creatinine & $1.07 \pm 2.64$ & $1.15 \pm 2.54$ \\
\hline Baseline GFR & $77.28 \pm 24.31$ & $79.71 \pm 23.09$ \\
\hline Post operative creatinine & $1.03 \pm 0.44$ & $1.19 \pm 0.49$ \\
\hline Post operative GFR & $71.02 \pm 22.81$ & $75.11 \pm 24.12$ \\
\hline
\end{tabular}

Table S2: The demographical properties of the populations allocated to the training and validation cohorts.

\author{
Abbreviations \\ Partial nephrectomy - PN. \\ Acute kidney injury - AKI. \\ Chronic kidney disease - CKD. \\ Machine learning $-M L$. \\ Random forest - RF. \\ Decision tree - DT. \\ Boolean satisfaction problem - SAT. \\ Receiver operation characteristic - ROC. \\ Area under the ROC curve - AUC. \\ Warm ischemia time - WIT. \\ K-nearest neighbors - KNN.
}

\title{
Declarations
}

\section{Ethics approval and consent to participate}

This research study was conducted retrospectively from data obtained for clinical purposes.

We consulted extensively with the Bnai Zion Medical Center who provided our study approval numbered BZ-0049-10.

\section{Consent to Publication}

Informed consent was obtained from all individual participants included in the study. 


\section{Availability of data and material}

All the data that has been used is available in the code repository of the project in Github. Upon acceptance, we will publish all the code used in a GitHub repository. The final outcome can be reviewed at https://teddy4445.github.io/cancer-AKI-predictor-

gui/predictor.html

\section{Competing interests}

The authors have no conflicts of interest to declare that are relevant to the content of this article.

\section{Funding}

No funds, grants, or other support were received.

\section{Author's Contribution}

T.L.: Project development, Data analysis, Manuscript writing. Z.B.: Project development, Data collection, Manuscript writing. S.B-M.: Manuscript editing. S.H.: Data management, Data collection, Manuscript editing.

All the authors have read and approved the final manuscript.

\section{Acknowledgments}

Not applicable.

\section{References}

[1] Ferlay, J., Colombet, M., Soerjomataram, I., et al., Cancer incidence and mortality patterns in Europe: Estimates for 40 countries and 25 major cancers in 2018. Eur J Cancer, 2018; 103: 356.

[2] Chow W.H., Devesa S.S., Contemporary epidemiology of renal cell cancer. Cancer J. 2008; 14: $288-301$.

[3] EAU, url: https://uroweb.org/guidelines/ (13.12.2020) 
[4] Scosyrev, E., Messing, E. M., Sylvester, R., et al., Renal function after nephron-sparing surgery versus radical nephrectomy: results from EORTC randomized trial 30904. Eur Urol, 2014; 65: 372.

[5] Bravi, C. A., Vertosick, E., Benfante, N., et al., Impact of Acute Kidney Injury and Its Duration on Long-term Renal Function After Partial Nephrectomy. Eur Urol. 2019; 76(3):

398-403.

[6] Zabell, J., Isharwal, S., Dong, W., et al., Acute Kidney Injury after Partial Nephrectomy of SolitaryKidneys: Impact on Long-Term Stability of Renal Function. J Urol. 2018; 200(6): 1295-

1301.

[7] Patel, H. D., Pierorazio, P. M., Johnson, M. H., et al., Renal Functional Outcomes after Surgery, Ablation, and Active Surveillance of Localized Renal Tumors: A Systematic Review and Meta-Analysis. American society of Nephrology. 2017; 15, 1555-9041.

[8] Shah, P., Kendall, F., Khozin, S, et al., Artificial intelligence and machine learning in clinical development:a transnational perspective. Npj Digit. Med. 2019; 2, 69.

[9] Boyko, N., Sviridova, T., Shakhovska, N., Use of machine learning in the forecast of clinical consequences of cancer diseases. 7th Mediterranean Conference on Embedded

Computing (MECO). 2018; 1-6.

[10] Weng, S. F., Reps, J., Kai, J., et al., Can machine-learning improve cardiovascular risk prediction using routine clinical data?. PLOS ONE. 2017; 12, e0174944.

[11] Wu, C. C., Yeh, W. C., Hsu, W. D., et al., Prediction of fatty liver disease using machine learning algorithms, Computer Methods, and Programs in Biomedicine. 2019; 170, 23-29. doi: 10.1016/j.cmpb.2018.12.032

[12] Thottakkara, P., Ozrazgat-Baslanti, T., Hupf, B. B., et al., Application of machine learning techniques tohigh-dimensional clinical data to forecast postoperative complications. PLoS

ONE. 2016; 11: e0155705.

[13] Emmert-Streib, F., Dehmer, M., A Machine Learning Perspective on Personalized Medicine: An Automated, Comprehensive Knowledge Base with Ontology for Pattern Recognition. Machine Learning andKnowledge Extraction. 2018; 1: 149-156.

[14] Flechet, M., G uiza, F., Schetz, M., et al., AKIpredictor, an online prognostic calculator for acute kidney injury in adult critically ill patients: development, validation, and comparison to serum neutrophilgelatinase-associated lipocalin. Intensive Care Med. 2017; 43(6): 764-773.

[15] Bellomo, R., Ronco, C., Kellum, J.A. et al., Acute renal failure - definition, outcome measures, animal models, fluid therapy and information technology needs: the Second International Consensus Conference of the Acute Dialysis Quality Initiative (ADQI) Group.

Crit Care 8. 2004.

[16] Mehta, R.L., Kellum, J.A., Shah, S.V. et al., Acute Kidney Injury Network: report of an initiative to improve outcomes in acute kidney injury. Crit Care 11, R31, 2007. 
[17] Bahouth, Z., Halachmi, S., Getzler, I., et al.. Functional and oncological outcomes of open nephron-sparing surgery for complex renal masses. Urol Oncol Semin Orig Investig.

2015; 33: 427.

[18] Mangasarian, O. L., Setiono, R., Wolberg, W. H., Pattern recognition via linear programming: Theory and application to medical diagnosis. Large-scale numerical optimization. 1990; 22-30.

[19] Breiman L., Random Forests. Machine Learning. 2001; 45: 5-32.

[20] Guyon, I., Cawley, G., An improved Random Forests approach with application to the performance pre-diction challenge datasets. 2009; 1. doi: 10.1.1.546.9501

[21] Liu, R., Liu, E., Yang, J., et al., Optimizing the Hyper-parameters for SVM by Combining Evolution Strategies with a Grid Search. Intelligent Control and Automation. Lecture Notes in Control and InformationSciences, Springer, Berlin, Heidelberg. 2006; 344.

[22] Wu, G., Xu, J., Optimized Approach of Feature Selection Based on Information Gain. International Conference on Computer Science and Mechanical Automation (CSMA), Hangzhou. 2015; 157-161, doi: 10.1109/C-SMA.2015.38.

[23] Coca, S. G., Yusuf, B., Shlipak, M. G., et al., Long-term risk of mortality and other adverse outcomes after acute kidney injury: a systematic review and meta-analysis. Am J Kidney Dis, 2009; 53(6): 961-973.

[24] Hidekazu, T., Tsunenori, K., Kazuhiko, Y., et al., Lower Incidence of Postoperative Acute Kidney Injury in Robot-Assisted Partial Nephrectomy Than in Open Partial Nephrectomy: A Propensity Score-MatchedStudy. Journal of Endourology. 2020; 34(7), 754-762.

[25] Slaqm, M., Shafique, I. B., Rahman, K. et al., A Simple Study on Weight and Height of Students. European Scientific Journal. 2017; 13: 63-71.

[26] Sokol, K., Flach, P., Desiderata for Interpretability: Explaining Decision Tree Predictions with Counter-factuals. AAAI Conference on Artificial Intelligence (AAAI-19). 2019; 10035-

10036.

[27] Cruz, D.N., de Cal, M., Garzotto, F., et al., Plasma neutrophil gelatinase-associated lipocalin is an early biomarker for acute kidney injury in an adult ICU population. Intensive

Care Med. 2010; 36: 444-451.

[28] Rank, N., Pfahringer, B., Kempfert, J., et al., Deep-learning-based real-time prediction of acute kidney injury outperforms human predictive performance. npj Digit. Med. 2020; 3:

139.

[29] Batchman, L. E., Foster, C. G., Missile system incorporating a targeting aid for man-inthe-loop missile controller. U.S. Patent 605307A. Issued February 25, 1997.

[30] Mehta, R. L., Cerda, J., Burdmann, E. A., et al., International Society of Nephrology's Oby 25 initiative for acute kidney injury (zero preventable deaths by 2025): a human rights case for nephrology. The Lancet Commissions. 2015; 385(9987): 2616-2643. 